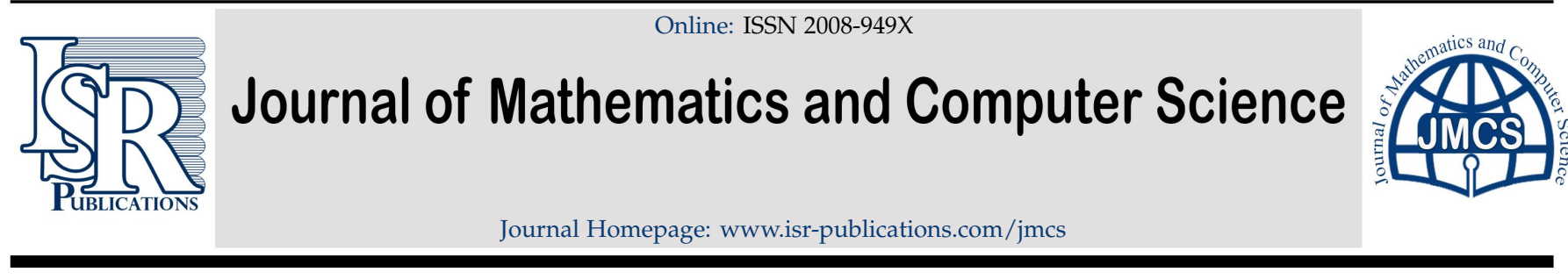

\title{
On approximate solutions for fractional system of differen- tial equations with Caputo-Fabrizio fractional operator
}

\author{
Hassan Kamil Jassim*, Mohammed Abed Shareef Hussain \\ Department of Mathematicas, Faculty of Education for Pure Sciences, University of Thi-Qar, Nasiriyah, Iraq.
}

\begin{abstract}
In this paper, we apply the Daftardar-Jafari method (DJM) and Sumudu decomposition method (SDM) to obtain the approximate analytical solutions of the fractional system of differential equations with Caputo-Fabrizio fractional derivative operator. The results obtained by DJM are compared with the results obtained by SDM. The results reveal that the suggested algorithms are very effective and simple and can be applied for linear and nonlinear problems in sciences and engineering.
\end{abstract}

Keywords: Daftardar-Jafari method, Sumudu transform, Adomian decomposition method, Caputo-Fabrizio fractional operator. 2020 MSC: 26A33, 34A08, 35R11.

(C)2021 All rights reserved.

\section{Introduction}

The concept of fractional calculus can be traced back to the letter of Leibniz to LHospital in 1695. Mathematician Euler discovered the gamma function in 1729, which established the foundation of fractional derivative. Compared with the classical integer-order models, fractional derivatives provide a more profound and comprehensive explanation about memory, heredity, non-locality of complex phenomena and processes. At present, the most commonly used definitions of fractional derivatives are the GrunwaldLetnikov derivative, the Riemann-Liouville derivative, the Riesz derivative, and the Caputo derivative [22]. Some new fractional derivatives, such as the Caputo-Fabrizio derivative and the Atangana-Baleanu fractional derivative, have tremendously promoted the capability of modeling complex physical phenomena and processes. The connatural behavior has been analyzed for evolution equations generated by three fractional derivatives namely the Riemann-Liouville, Caputo-Fabrizio, and Atangana-Baleanu fractional derivatives $[2,3,17]$.

Several analytical and numerical techniques were successfully applied to deal with fractional partial differential equations, the homotopy perturbation method [10, 23], the series expansion method [18, 25], Adomian decomposition methods [5,27], the variational iteration method [16, 20], reduce differential transform method [12, 14], homotopy perturbation transform methods $[4,9,21]$, and other analytical approaches that could be of interest for the reader are presented in $[6,7,11,13,15,19,24,26]$.

\footnotetext{
${ }^{*}$ Corresponding author

Email addresses: hassankamil@utq.edu.iq (Hassan Kamil Jassim), mshirq@gmail .com (Mohammed Abed Shareef Hussain)

doi: $10.22436 /$ jmcs.023.01.06
}

Received: 2020-01-21 Revised: 2020-05-15 Accepted: 2020-08-17 
In this paper, we use the DJM and SDM to solve nonlinear fractional system of partial differential equations involving the fractional operator of Caputo-Fabrizio type. The paper has been organized as follows. The basic definitions of fractional calculus are given in Section 2, analysis of the methods used is given in Section 3 and Section 4, An illustrative example that show the effectiveness of the proposed method is given in Section 5, and finally the conclusion is given in Section 6.

\section{Preliminaries of fractional calculus}

Definition 2.1 ([1, 8, 9, 24]). The Caputo-Fabrizio fractional operator is defined for $n-1<\alpha \leqslant n$ as:

$$
{ }^{C F} D_{t}^{\alpha} u(t)=\frac{(2-\alpha) M(\alpha)}{2(1-\alpha)} \int_{0}^{t} \exp \left[-\frac{\alpha(t-s)}{1-\alpha}\right] u^{(n)}(s) d s, t \geqslant 0,
$$

where $M(\alpha)$ is a normalization function such that $M(0)=M(1)=1$ and $u^{(n)}$ is the derivative of integer $n$ order of $u, n=1,2, \ldots, \in N$.

The following are the basic properties of the operator ${ }^{C F} D_{t}^{\alpha}$ :

1. ${ }^{C F} D_{t}^{\alpha} u(t)=u(t)$, where $\alpha=0$;

2. ${ }^{C F} D_{t}^{\alpha}[u(t)+v(t)]={ }^{C F} D_{t}^{\alpha} u(t)+{ }^{C F} D_{t}^{\alpha} v(t)$;

3. ${ }^{C F} D_{t}^{\alpha}(c)=0$, where $c$ is constant.

Definition $2.2([1,8])$. The fractional Caputo-Fabrizio fractional integral operator of order $0<\alpha \leqslant 1$ and $t>0$ is given by:

$$
{ }^{C F} I_{t}^{\alpha} u(t)=\frac{2(1-\alpha)}{(2-\alpha) M(\alpha)} u(t)+\frac{2 \alpha}{(2-\alpha) M(\alpha)} \int_{0}^{t} u(s) d s
$$

The following are the basic properties of the operator ${ }^{\mathrm{CF}} \mathrm{I}_{\mathrm{t}}^{\alpha}$ :

1. ${ }^{C F} I_{t}^{\alpha} u(t)=u(t)$, where $\alpha=0$;

2. ${ }^{C F} I_{t}^{\alpha}[u(t)+v(t)]={ }^{C F} I_{t}^{\alpha} u(t)+{ }^{C F} I_{t}^{\alpha} v(t)$;

3. ${ }^{C F} I_{t}^{\alpha}\left[{ }^{C F} D_{t}^{\alpha} u(t)\right]=u(t)-u(0)$.

Definition 2.3. The Sumudu transform is defined over the set of function

$$
A=\left\{u(t)\left|\exists M, \tau_{1}, \tau_{2}>0,\right| u(t) \mid<M e^{|t| / \tau_{j}}, \text { if } t \in(-1)^{j} \times[0, \infty)\right\},
$$

by the following formula

$$
\mathbf{S}\{\mathrm{u}(\mathrm{t})\}=\int_{0}^{\infty} \mathrm{u}(w \mathrm{t}) \mathrm{e}^{-\mathrm{t}} \mathrm{dt}, \quad w \in\left(\tau_{1}, \tau_{2}\right)
$$

\section{Analysis of DJ method}

Let us consider the following nonlinear partial differential equation in the Caputo-Fabrizio sense:

$$
{ }^{C F} D_{t}^{n+\alpha} u(x, t)+R[u(x, t)]+N[u(x, t)]=g(x, t)
$$

with initial conditions

$$
\frac{\partial^{k} u(x, 0)}{\partial t^{k}}=\phi_{k}(x), k=0,1, \ldots, m-1,
$$

where ${ }_{0}^{C F} D_{t}^{n+\alpha} u(x, t)$ is Caputo-Fabrizio operator of $u(x, t), m-1<n+\alpha \leqslant m, m \in N$, $R$ is a linear operator, $\mathrm{N}$ is an nonlinear operator and $\mathrm{g}$ is a source term.

Taking integral of Caput-Fabrizio to both sides of (3.1) we get

$$
{ }^{C F} I_{t}^{\alpha}\left[{ }^{C F} D_{t}^{n+\alpha} u(x, t)\right]+{ }^{C F} I_{t}^{\alpha} R[u(x, t)]+{ }^{C F} I_{t}^{\alpha} N[u(x, t)]={ }^{C F} I_{t}^{\alpha}[g(x, t)] .
$$


Then, we obtain

$$
u(x, t)=\sum_{k=0}^{m-1} u^{(k)}(x, 0) \frac{x^{k}}{k !}+{ }^{C F} I_{t}^{\alpha}[g(x, t)]-{ }^{C F} I_{t}^{\alpha} R[u(x, t)]-{ }^{C F} I_{t}^{\alpha} N[u(x, t)] .
$$

We are looking for a solution $u(x, t)$ of Eq. (3.2) having the series form:

$$
u(x, t)=\sum_{n=0}^{\infty} u_{n}(x, t)
$$

The non-linear operator $\mathrm{N}$ can be decomposed as

$$
N\left[\sum_{n=0}^{\infty} u_{n}(x, t)\right]=N\left[u_{0}\right]+\sum_{n=1}^{\infty}\left(N\left[\sum_{i=0}^{n} u_{i}\right]-N\left[\sum_{i=0}^{n-1} u_{i}\right]\right)
$$

In view of (3.3) and (3.4), Eq. (3.2) is equivalent to

$$
\begin{aligned}
\sum_{n=0}^{\infty} u_{n}= & \sum_{k=0}^{m-1} u^{(k)}(x, 0) \frac{x^{k}}{k !}+{ }^{C F} I_{t}^{\alpha}[g(x, t)]-{ }^{C F} I_{t}^{\alpha} R\left[\sum_{n=0}^{\infty} u_{n}\right] \\
& -{ }^{C F} I_{t}^{\alpha} N\left[u_{o}(x, t)\right]-{ }^{C F} I_{t}^{\alpha}\left(\sum_{n=1}^{\infty} N\left[\sum_{i=0}^{n} u_{i}\right]-N\left[\sum_{i=0}^{n-1} u_{i}\right]\right) .
\end{aligned}
$$

Moreover, the relation is defined with recurrence so that

$$
\begin{aligned}
u_{0}(x, t) & =\sum_{k=0}^{m-1} u^{(k)}(x, 0) \frac{x^{k}}{k !}+{ }^{C F} I_{t}^{\alpha}[g(x, t)], \\
u_{1}(x, t) & =-{ }^{C F} I_{t}^{\alpha} R\left[u_{0}\right]-{ }^{C F} I_{t}^{\alpha} N\left[u_{o}(x, t)\right], \\
u_{n+1}(x, t) & =-{ }^{C F} I_{t}^{\alpha} R\left[u_{n}\right]-{ }^{C F} I_{t}^{\alpha}\left(N\left[\sum_{i=0}^{n} u_{i}\right]-N\left[\sum_{i=0}^{n-1} u_{i}\right]\right), n=1,2, \ldots
\end{aligned}
$$

Then k-term approximate solution of Eq. (3.1) is given by:

$$
u(x, t)=u_{0}(x, t)+u_{1}(x, t)+u_{2}(x, t)+u_{3}(x, t)+\cdots
$$

\section{Analysis of Sumudu decomposition method}

Applying the Sumudu transform to (3.1) yields

$$
\mathbf{S}\left\{{ }^{\mathrm{CF}} \mathrm{D}_{\mathrm{t}}^{\mathrm{n}+\alpha} \mathfrak{u}(\mathrm{x}, \mathrm{t})\right\}+\mathbf{S}\{\mathrm{R}[\mathrm{u}(\mathrm{x}, \mathrm{t})]\}+\mathbf{S}\{\mathrm{N}[\mathrm{u}(\mathrm{x}, \mathrm{t})]\}=\mathbf{S}\{\mathrm{g}(\mathrm{x}, \mathrm{t})\} .
$$

By applying the Sumudu transform differentiation property, we have

$$
\frac{1}{(1-\alpha+\alpha \sigma)\left(\sigma^{\mathfrak{m}}\right)}\left[\mathbf{S}\{\mathfrak{u}(x, t)\}-\sum_{k=0}^{m-1} w^{k} \phi_{k}(x)\right]=\mathbf{S}\{g(x, t)\}-\mathbf{S}\{\mathbf{R}[\mathbf{u}(x, t)]\}-\mathbf{S}\{\mathbf{N}[\mathbf{u}(x, t)]\}
$$

or

$$
\begin{aligned}
\mathbf{S}\{\mathfrak{u}(x, t)\}= & \sum_{k=0}^{\mathrm{m}-1} w^{k} \phi_{k}(x)+(1-\alpha+\alpha \sigma) \sigma^{m} \mathbf{S}\{g(x, t)\} \\
& -(1-\alpha+\alpha \sigma) \sigma^{m} \mathbf{S}\{R[u(x, t)]\}-(1-\alpha+\alpha \sigma) \sigma^{m} \mathbf{S}\{N[u(x, t)]\} .
\end{aligned}
$$


Taking the inverse of Sumudu transform of (4.1), we obtain

$$
\begin{aligned}
u(x, t)= & \mathbf{S}^{-1}\left(\sum_{k=0}^{m-1} w^{k} \phi_{k}(x)\right)+\mathbf{S}^{-1}\left[(1-\alpha+\alpha \sigma) \sigma^{m} \mathbf{S}\{g(x, t)\}\right] \\
& -\mathbf{S}^{-1}\left[(1-\alpha+\alpha \sigma) \sigma^{m} \mathbf{S}\{R[u(x, t)]\}\right]-\mathbf{S}^{-1}\left[(1-\alpha+\alpha \sigma) \sigma^{m} \mathbf{S}\{\mathbf{N}[u(x, t)]\}\right] .
\end{aligned}
$$

Suppose that $u(x, t)$ is a solution of (4.2), which we express as

$$
u(x, t)=\sum_{n=0}^{\infty} u_{n}(x, t),
$$

the nonlinear term can be decomposed as

$$
\mathrm{N}[\mathrm{u}(\mathrm{x}, \mathrm{t})]=\sum_{\mathrm{n}=0}^{\infty} A_{n},
$$

where

$$
A_{n}=\frac{1}{n !} \frac{\partial^{n}}{\partial \xi^{n}}\left[N\left(\sum_{i=0}^{n} \xi^{i} u_{i}(x, t)\right)\right]_{\xi=0}, n=0,1,2, \ldots
$$

Substituting (4.3) and (4.4) into (4.2) gives us the result that

$$
\begin{aligned}
\mathfrak{u}(x, t)= & \mathbf{S}^{-\mathbf{1}}\left(\sum_{k=0}^{\mathrm{m}-1} w^{k} \phi_{k}(x)\right)+\mathbf{S}^{-\mathbf{1}}\left[(1-\alpha+\alpha \sigma) \sigma^{\mathrm{m}} \mathbf{S}\{g(x, t)\}\right] \\
& -\mathbf{S}^{-\mathbf{1}}\left[(1-\alpha+\alpha \sigma) \sigma^{\mathrm{m}} \mathbf{S}\left\{\mathrm{R}\left[\sum_{n=0}^{\infty} u_{n}(x, t)\right]\right\}\right]-\mathbf{S}^{-\mathbf{1}}\left[(1-\alpha+\alpha \sigma) \sigma^{\mathrm{m}} \mathbf{S}\left\{\sum_{n=0}^{\infty} A_{n}\right\}\right] .
\end{aligned}
$$

When we compare the left and right hand sides of (4.5) we obtain

$$
\begin{aligned}
& \mathfrak{u}_{0}(x, t)=\mathbf{S}^{-1}\left(\sum_{k=0}^{m-1} w^{k} \phi_{k}(x)\right)+\mathbf{S}^{-1}\left[(1-\alpha+\alpha \sigma) \sigma^{m} \mathbf{S}\{g(x, t)\}\right], \\
& u_{1}(x, t)=-\mathbf{S}^{-1}\left[(1-\alpha+\alpha \sigma) \sigma^{m} \mathbf{S}\left\{R\left[u_{0}(x, t)\right]\right\}\right]-\mathbf{S}^{-1}\left[(1-\alpha+\alpha \sigma) \sigma^{m} \mathbf{S}\left\{A_{0}\right\}\right], \\
& \mathfrak{u}_{2}(x, t)=-\mathbf{S}^{-1}\left[(1-\alpha+\alpha \sigma) \sigma^{m} \mathbf{S}\left\{R\left[u_{1}(x, t)\right]\right\}\right]-\mathbf{S}^{-1}\left[(1-\alpha+\alpha \sigma) \sigma^{m} \mathbf{S}\left\{A_{1}\right\}\right],
\end{aligned}
$$

The recursive relation in its general form is

$$
\begin{aligned}
& u_{0}(x, t)=S^{-1}\left(\sum_{k=0}^{m-1} w^{k} \phi_{k}(x)\right)+S^{-1}\left[(1-\alpha+\alpha \sigma) \sigma^{m} \mathbf{S}\{g(x, t)\}\right], \\
& u_{n}(x, t)=-S^{-1}\left[(1-\alpha+\alpha \sigma) \sigma^{m} \mathbf{S}\left\{R\left[u_{n-1}(x, t)\right]\right\}\right]-S^{-1}\left[(1-\alpha+\alpha \sigma) \sigma^{m} \mathbf{S}\left\{A_{n-1}\right\}\right] .
\end{aligned}
$$

The approximate solution is given by

$$
u(x, t)=u_{0}(x, t)+u_{2}(x, t)+u_{3}(x, t)+\cdots .
$$

\section{Illustrative paradigm}

Example 1. Consider the nonlinear system of time-fractional differential equation in the Caputo-Fabrizio operator:

$$
\begin{aligned}
& { }^{C F} D_{t}^{\alpha}(x, y, t)=-u+v_{y} w_{x}-w_{y} v_{x} \\
& { }^{C F} D_{t}^{\beta}(x, y, t)=v-u_{y} w_{x}-w_{y} u_{x}, \\
& { }^{C F} D_{t}^{\varepsilon}(x, y, t)=w-v_{y} u_{x}-u_{y} v_{x}
\end{aligned}
$$

where $0<\alpha, \beta, \varepsilon \leqslant 1$ and the initial conditions are 


$$
u(x, y, 0)=e^{x+y}, \quad v(x, y, 0)=e^{x-y}, \quad w(x, y, 0)=e^{y-x} .
$$

Below we present the DJM.

Taking ${ }^{C F} \mathrm{I}_{t}^{\alpha},{ }^{C F} I_{t}^{\beta}$, and ${ }^{C F} I_{t}^{\varepsilon}$ to both sides of (5.1), respectively, we get

$$
\begin{gathered}
u(x, y, t)=u(x, y, 0)-{ }^{C F} I_{t}^{\alpha}[u]+{ }^{C F} I_{t}^{\alpha}\left[\frac{\partial v}{\partial y} \frac{\partial w}{\partial x}-\frac{\partial w}{\partial y} \frac{\partial v}{\partial x}\right], \\
v(x, y, t)=v(x, y, 0)+{ }^{C F} I_{t}^{\beta}[v]-{ }^{C F} I_{t}^{\beta}\left[\frac{\partial u}{\partial y} \frac{\partial w}{\partial x}+\frac{\partial w}{\partial y} \frac{\partial u}{\partial x}\right], \\
w(x, y, t)=w(x, y, 0)+{ }^{C F} I_{t}^{\varepsilon}[w]-{ }^{C F} I_{t}^{\varepsilon}\left[\frac{\partial v}{\partial y} \frac{\partial u}{\partial x}+\frac{\partial u}{\partial y} \frac{\partial v}{\partial x}\right] .
\end{gathered}
$$

Thus according to Eq. (3.5), the approximate solution can be obtained:

$$
\begin{aligned}
u_{0}(x, y, t)= & u(x, y, 0), \quad v_{0}(x, y, t)=v(x, y, 0), \quad w_{0}(x, y, t)=w(x, y, 0), \\
u_{1}(x, y, t)= & -{ }^{C F} I_{t}^{\alpha}\left[u_{0}\right]+{ }^{C F} I_{t}^{\alpha}\left[\frac{\partial v_{0}}{\partial y} \frac{\partial w_{0}}{\partial x}-\frac{\partial w_{0}}{\partial y} \frac{\partial v_{0}}{\partial x}\right], \\
v_{1}(x, y, t)= & { }^{C F} I_{t}^{\beta}\left[v_{0}\right]-{ }^{C F} I_{t}^{\beta}\left[\frac{\partial u_{0}}{\partial y} \frac{\partial w_{0}}{\partial x}+\frac{\partial w_{0}}{\partial y} \frac{\partial u_{0}}{\partial x}\right], \\
w_{1}(x, y, t)= & { }^{C F} I_{t}^{\varepsilon}\left[w_{0}\right]-{ }^{C F} I_{t}^{\varepsilon}\left[\frac{\partial v_{0}}{\partial y} \frac{\partial u_{0}}{\partial x}+\frac{\partial u_{0}}{\partial y} \frac{\partial v_{0}}{\partial x}\right], \\
u_{2}(x, y, t)= & -{ }^{C F} I_{t}^{\alpha}\left[u_{1}\right]+{ }^{C F} I_{t}^{\alpha}\left[\frac{\partial\left(v_{0}+v_{1}\right)}{\partial y} \frac{\partial\left(w_{0}+w_{1}\right)}{\partial x}-\frac{\partial v_{0}}{\partial y} \frac{\partial w_{0}}{\partial x}\right] \\
& -{ }^{C F} I_{t}^{\alpha}\left[\frac{\partial\left(w_{0}+w_{1}\right)}{\partial y} \frac{\partial\left(v_{0}+v_{1}\right)}{\partial x}+\frac{\partial w_{0}}{\partial y} \frac{\partial v_{0}}{\partial x}\right] \\
v_{2}(x, y, t)= & { }^{C F} I_{t}^{\beta}\left[v_{1}\right]-{ }^{C F} I_{t}^{\beta}\left[\frac{\partial\left(u_{0}+u_{1}\right)}{\partial y} \frac{\partial\left(w_{0}+w_{1}\right)}{\partial x}-\frac{\partial u_{0}}{\partial y} \frac{\partial w_{0}}{\partial x}\right] \\
& +{ }^{C F} I_{t}^{\beta}\left[\frac{\partial\left(w_{0}+w_{1}\right)}{\partial y} \frac{\partial\left(u_{0}+u_{1}\right)}{\partial x}+\frac{\partial w_{0}}{\partial y} \frac{\partial u_{0}}{\partial x}\right] \\
w_{2}(x, y, t)= & { }^{C F} I_{t}^{\varepsilon}\left[w_{1}\right]-{ }^{C F} I_{t}^{\varepsilon}\left[\frac{\partial\left(v_{0}+v_{1}\right)}{\partial y} \frac{\partial\left(u_{0}+u_{1}\right)}{\partial x}-\frac{\partial v_{0}}{\partial y} \frac{\partial u_{0}}{\partial x}\right] \\
& -{ }^{C F} I_{t}^{\varepsilon}\left[\frac{\partial\left(u_{0}+u_{1}\right)}{\partial y} \frac{\partial\left(v_{0}+v_{1}\right)}{\partial x}+\frac{\partial u_{0}}{\partial y} \frac{\partial v_{0}}{\partial x}\right]
\end{aligned}
$$

By the above algorithms, we obtain:

$$
\begin{aligned}
\mathrm{u}_{0}(x, y, t)= & e^{x+y}, \quad v_{0}(x, y, t)=e^{x-y}, \quad w_{0}(x, y, t)=e^{y-x}, \\
u_{1}(x, y, t)= & -{ }^{C F} I_{t}^{\alpha}\left[e^{x+y}\right]+{ }^{C F} I_{t}^{\alpha}\left[e^{x-y} e^{y-x}-e^{y-x} e^{x-y}\right]=-e^{x+y}(1-\alpha+\alpha t), \\
v_{1}(x, y, t)= & { }^{C F} I_{t}^{\beta}\left[e^{x-y}\right]-{ }^{C F} I_{t}^{\beta}\left[-e^{x+y} e^{y-x}+e^{y-x} e^{x+y}\right]=e^{x-y}(1-\beta+\beta t), \\
w_{1}(x, y, t)= & { }^{C F} I_{t}^{\varepsilon}\left[e^{x-y}\right]-{ }^{C F} I_{t}^{\varepsilon}\left[-e^{x+y} e^{x-y}+e^{x-y} e^{x+y}\right]=e^{y-x}(1-\varepsilon+\varepsilon t), \\
u_{2}(x, y, t)= & -{ }^{C F} I_{t}^{\alpha}\left[-e^{x+y}(1-\alpha+\alpha t)\right]+{ }^{C F} I_{t}^{\alpha}[(2-\beta+\beta t)(2-\varepsilon+\varepsilon t)-(2-\varepsilon+\varepsilon t)(2-\beta+\beta t)] \\
& -{ }^{C F} I_{t}^{\alpha}\left[e^{x-y} e^{y-x}-e^{y-x} e^{x-y}\right] \\
= & (1-\alpha) e^{x+y}(1-\alpha+\alpha t)+\alpha e^{x+y}\left(t-\alpha t+\frac{1}{2} \alpha t^{2}\right), \\
v_{2}(x, y, t)= & { }^{C F} I_{t}^{\beta}\left[e^{x-y}(1-\beta+\beta t)\right]-{ }^{C F} I_{t}^{\beta}[-(\alpha t-\alpha)(2-\varepsilon+\varepsilon t)+(2-\varepsilon+\varepsilon t)(\alpha t-\alpha)]
\end{aligned}
$$




$$
\begin{aligned}
& +{ }^{C F} I_{t}^{\beta}\left[-e^{x+y} e^{y-x}+e^{y-x} e^{x+y}\right] \\
= & (1-\beta) e^{x-y}(1-\beta+\beta t)+\beta e^{x-y}\left(t-\beta t+\frac{1}{2} \beta t^{2}\right), \\
w_{2}(x, y, t)= & { }^{C F} I_{t}^{\varepsilon}\left[e^{y-x}(1-\varepsilon+\varepsilon t)\right]-{ }^{C F} I_{t}^{\varepsilon}[-(\alpha t-\alpha)(2-\beta+\beta t)+(2-\beta+\beta t)(\alpha t-\alpha)] \\
& +{ }^{C F} I_{t}^{\varepsilon}\left[-e^{x+y} e^{x-y}+e^{x-y} e^{x+y}\right] \\
= & (1-\varepsilon) e^{y-x}(1-\varepsilon+\varepsilon t)+\varepsilon e^{y-x}\left(t-\varepsilon t+\frac{1}{2} \varepsilon t^{2}\right)
\end{aligned}
$$

Then the approximate solution of (5.1) is

$$
\begin{aligned}
& u(x, y, t)=e^{x+y}-e^{x+y}(1-\alpha+\alpha t)+(1-\alpha) e^{x+y}(1-\alpha+\alpha t)+\alpha e^{x+y}\left(t-\alpha t+\frac{1}{2} \alpha t^{2}\right)+\cdots, \\
& v(x, y, t)=e^{x-y}+e^{x-y}(1-\beta+\beta t)+(1-\beta) e^{x-y}(1-\beta+\beta t)+\beta e^{x-y}\left(t-\beta t+\frac{1}{2} \beta t^{2}\right)+\cdots, \\
& w(x, y, t)=e^{y-x}+e^{y-x}(1-\varepsilon+\varepsilon t)+(1-\varepsilon) e^{y-x}(1-\varepsilon+\varepsilon t)+\varepsilon e^{y-x}\left(t-\varepsilon t+\frac{1}{2} \varepsilon t^{2}\right)+\cdots .
\end{aligned}
$$

As a next step, we apply the SADM. Taking the Sumudu transform on both sides of (5.1), we have

$$
\begin{aligned}
& \mathbf{S}\{\mathfrak{u}(x, y, t)\}=u(x, y, 0)+(1-\alpha+\alpha \sigma) \mathbf{S}\left\{-\mathfrak{u}+\frac{\partial v}{\partial y} \frac{\partial w}{\partial x}-\frac{\partial w}{\partial y} \frac{\partial v}{\partial x}\right\}, \\
& \mathbf{S}\{v(x, y, t)\}=v(x, y, 0)+(1-\beta+\beta \sigma) \mathbf{S}\left\{v-\frac{\partial u}{\partial y} \frac{\partial w}{\partial x}-\frac{\partial w}{\partial y} \frac{\partial u}{\partial x}\right\}, \\
& \mathbf{S}\{w(x, y, t)\}=w(x, y, 0)+(1-\varepsilon+\varepsilon \sigma) \mathbf{S}\left\{w-\frac{\partial v}{\partial y} \frac{\partial u}{\partial x}-\frac{\partial u}{\partial y} \frac{\partial v}{\partial x}\right\},
\end{aligned}
$$

or

$$
\begin{aligned}
& \mathbf{S}\{u(x, y, t)\}=e^{x+y}+(1-\alpha+\alpha \sigma) \mathbf{S}\left\{-u+\frac{\partial v}{\partial y} \frac{\partial w}{\partial x}-\frac{\partial w}{\partial y} \frac{\partial v}{\partial x}\right\}, \\
& \mathbf{S}\{v(x, y, t)\}=e^{x-y}+(1-\beta+\beta \sigma) \mathbf{S}\left\{v-\frac{\partial u}{\partial y} \frac{\partial w}{\partial x}-\frac{\partial w}{\partial y} \frac{\partial u}{\partial x}\right\}, \\
& \mathbf{S}\{w(x, y, t)\}=e^{y-x}+(1-\varepsilon+\varepsilon \sigma) \mathbf{S}\left\{w-\frac{\partial v}{\partial y} \frac{\partial u}{\partial x}-\frac{\partial u}{\partial y} \frac{\partial v}{\partial x}\right\} .
\end{aligned}
$$

Operating with the ST on both sides of (5.3) gives

$$
\begin{aligned}
& u(x, y, t)=e^{x+y}+\mathbf{S}^{-1}\left[(1-\alpha+\alpha \sigma) \mathbf{S}\left\{-\mathfrak{u}+\frac{\partial v}{\partial y} \frac{\partial w}{\partial x}-\frac{\partial w}{\partial y} \frac{\partial v}{\partial x}\right\}\right] \\
& v(x, y, t)=e^{x-y}+\mathbf{S}^{-1}\left[(1-\beta+\beta \sigma) \mathbf{S}\left\{v-\frac{\partial u}{\partial y} \frac{\partial w}{\partial x}-\frac{\partial w}{\partial y} \frac{\partial u}{\partial x}\right\}\right] \\
& w(x, y, t)=e^{y-x}+\mathbf{S}^{-1}\left[(1-\varepsilon+\varepsilon \sigma) S\left\{w-\frac{\partial v}{\partial y} \frac{\partial u}{\partial x}-\frac{\partial u}{\partial y} \frac{\partial v}{\partial x}\right\}\right]
\end{aligned}
$$

Now, we represent solution as an infinite series given below

$$
u(x, y, t)=\sum_{n=0}^{\infty} u_{n}(x, y, t), \quad v(x, y, t)=\sum_{n=0}^{\infty} v_{n}(x, y, t), \quad w(x, y, t)=\sum_{n=0}^{\infty} w_{n}(x, y, t),
$$


and the nonlinear terms can be decomposed as

$$
v_{y} w_{x}-w_{y} v_{x}=\sum_{n=0}^{\infty} A_{n}, \quad u_{y} w_{x}-w_{y} u_{x}=\sum_{n=0}^{\infty} B_{n}, \quad v_{y} u_{x}-u_{y} v_{x}=\sum_{n=0}^{\infty} C_{n}
$$

where

$$
\begin{aligned}
\mathrm{A}_{0} & =\frac{\partial v_{0}}{\partial y} \frac{\partial w_{0}}{\partial x}-\frac{\partial w_{0}}{\partial y_{0}} \frac{\partial v_{0}}{\partial x} \\
\mathrm{~A}_{1} & =\frac{\partial v_{0}}{\partial y} \frac{\partial w_{1}}{\partial x}+\frac{\partial v_{1}}{\partial y} \frac{\partial w_{0}}{\partial x}-\frac{\partial w_{0}}{\partial y} \frac{\partial v_{1}}{\partial x}-\frac{\partial w_{1}}{\partial y} \frac{\partial v_{0}}{\partial x} \\
& \vdots \\
\mathrm{B}_{0} & =-\frac{\partial \mathrm{u}_{0}}{\partial y} \frac{\partial w_{0}}{\partial x}-\frac{\partial w_{0}}{\partial y} \frac{\partial u_{0}}{\partial x} \\
\mathrm{~B}_{1} & =-\frac{\partial \mathrm{u}_{0}}{\partial y} \frac{\partial w_{1}}{\partial x}-\frac{\partial \mathrm{u}_{1}}{\partial y} \frac{\partial w_{0}}{\partial x}-\frac{\partial w_{0}}{\partial y} \frac{\partial u_{1}}{\partial x}-\frac{\partial w_{1}}{\partial y} \frac{\partial u_{0}}{\partial x} \\
& \vdots \\
\mathrm{C}_{0} & =-\frac{\partial v_{0}}{\partial y} \frac{\partial u_{0}}{\partial x}-\frac{\partial u_{0}}{\partial y} \frac{\partial v}{\partial x} \\
\mathrm{C}_{1} & =-\frac{\partial v_{0}}{\partial y} \frac{\partial u_{1}}{\partial x}-\frac{\partial v_{1}}{\partial y} \frac{\partial u_{0}}{\partial x}-\frac{\partial u_{0}}{\partial y} \frac{\partial v_{1}}{\partial x}-\frac{\partial u_{1}}{\partial y} \frac{\partial v_{0}}{\partial x}
\end{aligned}
$$

Substituting (5.5) and (5.6) in (5.4), we get

$$
\begin{aligned}
& \sum_{n=0}^{\infty} u_{n}(x, y, t)=e^{x+y}+\mathbf{S}^{-1}\left[(1-\alpha+\alpha \sigma) \mathbf{S}\left\{-\sum_{n=0}^{\infty} u_{n}+\sum_{n=0}^{\infty} A_{n}\right\}\right], \\
& \sum_{n=0}^{\infty} v_{n}(x, y, t)=e^{x-y}+\mathbf{S}^{-1}\left[(1-\beta+\beta \sigma) \mathbf{S}\left\{\sum_{n=0}^{\infty} v_{n}-\sum_{n=0}^{\infty} B_{n}\right\}\right], \\
& \sum_{n=0}^{\infty} w_{n}(x, y, t)=e^{y-x}+\mathbf{S}^{-1}\left[(1-\varepsilon+\varepsilon \sigma) \mathbf{S}\left\{\sum_{n=0}^{\infty} w_{n}-\sum_{n=0}^{\infty} C_{n}\right\}\right] .
\end{aligned}
$$

On comparing both sides of the (5.7), we get

$$
\begin{aligned}
u_{0}(x, y, t) & =e^{x+y}, \quad v_{0}(x, y, t)=e^{x-y}, \quad w_{0}(x, y, t)=e^{y-x}, \\
u_{1}(x, y, t) & =\mathbf{S}^{-1}\left[(1-\alpha+\alpha \sigma) \mathbf{S}\left\{-u_{0}+A_{0}\right\}\right] \\
& =\mathbf{S}^{-1}\left[(1-\alpha+\alpha \sigma) \mathbf{S}\left\{-e^{x+y}+\left(-e^{x-y} e^{y-x}+e^{x-y} e^{y-x}\right)\right\}\right] \\
& =\mathbf{S}^{-1}\left[(1-\alpha+\alpha \sigma) \mathbf{S}\left\{-e^{x+y}\right\}\right] \\
& =-e^{x+y} \mathbf{S}^{-1}[(1-\alpha+\alpha \sigma)]=-e^{x+y}(1-\alpha+\alpha t), \\
v_{1}(x, y, t) & =\mathbf{S}^{-1}\left[(1-\beta+\beta \sigma) \mathbf{S}\left\{v_{0}+B_{0}\right\}\right] \\
& =\mathbf{S}^{-1}\left[(1-\beta+\beta \sigma) \mathbf{S}\left\{e^{x-y}+\left(e^{x+y} e^{y-x}-e^{x+y} e^{y-x}\right)\right\}\right] \\
& =\mathbf{S}^{-1}\left[(1-\beta+\beta \sigma) \mathbf{S}\left\{e^{x-y}\right\}\right] \\
& =e^{x-y} \mathbf{S}^{-1}[(1-\beta+\beta \sigma)]=e^{x-y}(1-\beta+\beta t), \\
w_{1}(x, y, t) & =\mathbf{S}^{-1}\left[(1-\varepsilon+\varepsilon \sigma) \mathbf{S}\left\{w_{0}+C_{0}\right\}\right]
\end{aligned}
$$




$$
\begin{aligned}
& =\mathbf{S}^{-\mathbf{1}}\left[(1-\varepsilon+\varepsilon \sigma) \mathbf{S}\left\{e^{y-x}+\left(e^{x+y} e^{x-y}-e^{x+y} e^{x-y}\right)\right\}\right] \\
& =\mathbf{S}^{-1}\left[(1-\varepsilon+\varepsilon \sigma) \mathbf{S}\left\{e^{y-x}\right\}\right] \\
& =e^{y-x} \mathbf{S}^{-1}[(1-\varepsilon+\varepsilon \sigma)]=e^{y-x}(1-\varepsilon+\varepsilon t), \\
u_{2}(x, y, t) & =\mathbf{S}^{-1}\left[(1-\alpha+\alpha \sigma) \mathbf{S}\left\{-u_{1}+A_{1}\right\} \mathbf{S}^{-1}\right] \\
& =\mathbf{S}^{-\mathbf{1}}\left[(1-\alpha+\alpha \sigma) \mathbf{S}\left\{e^{x+y}(1-\alpha+\alpha t)\right\}\right] \\
& =e^{x+y} \mathbf{S}^{-1}\left[(1-\alpha+\alpha \sigma)^{2}\right]=e^{x+y}\left[\left(1-2 \alpha+\alpha^{2}\right)+\left(2 \alpha-2 \alpha^{2}\right) t+\frac{1}{2} \alpha^{2} t^{2}\right], \\
v_{2}(x, y, t) & =\mathbf{S}^{-1}\left[(1-\beta+\beta \sigma) \mathbf{S}\left\{v_{1}+B_{1}\right\}\right] \\
& =\mathbf{S}^{-\mathbf{1}}\left[(1-\beta+\beta \sigma) \mathbf{S}\left\{e^{x-y}(1-\beta+\beta t)\right\}\right] \\
& =e^{x-y} \mathbf{S}^{-1}\left[(1-\beta+\beta \sigma)^{2}\right]=e^{x-y}\left[\left(1-2 \beta+\beta^{2}\right)+\left(2 \beta-2 \beta^{2}\right) t+\frac{1}{2} \beta^{2} t^{2}\right], \\
w_{2}(x, y, t) & =\mathbf{S}^{-1}\left[(1-\varepsilon+\varepsilon \sigma) \mathbf{S}\left\{w_{1}+C_{1}\right\}\right] \\
& =\mathbf{S}^{-\mathbf{1}}\left[(1-\varepsilon+\varepsilon \sigma) \mathbf{S}\left\{e^{y-x}(1-\varepsilon+\varepsilon t)\right\}\right] \\
& =e^{y-x} \mathbf{S}^{-1}\left[(1-\varepsilon+\varepsilon \sigma)^{2}\right] \\
& =e^{y-x}\left[\left(1-2 \varepsilon+\varepsilon^{2}\right)+\left(2 \varepsilon-2 \varepsilon^{2}\right) t+\frac{1}{2} \varepsilon^{2} t^{2}\right] .
\end{aligned}
$$

Then the approximate solution of (5.1) is

$$
\begin{aligned}
& u(x, y, t)=e^{x+y}-e^{x+y}(1-\alpha+\alpha t)+e^{x+y}\left[\left(1-2 \alpha+\alpha^{2}\right)+\left(2 \alpha-2 \alpha^{2}\right) t+\frac{1}{2} \alpha^{2} t^{2}+\cdots\right], \\
& v(x, y, t)=e^{x-y}+e^{x-y}(1-\beta+\beta t)+e^{x-y}\left[\left(1-2 \beta+\beta^{2}\right)+\left(2 \beta-2 \beta^{2}\right) t+\frac{1}{2} \beta^{2} t^{2}+\cdots\right], \\
& w(x, y, t)=e^{y-x}+e^{y-x}(1-\varepsilon+\varepsilon t)+e^{y-x}\left[\left(1-2 \varepsilon+\varepsilon^{2}\right)+\left(2 \varepsilon-2 \varepsilon^{2}\right) t+\frac{1}{2} \varepsilon^{2} t^{2}+\cdots\right] .
\end{aligned}
$$

From Eqs. (5.2) and (5.8), the approximate solution of the given problem (5.1), by using DJM is the same result as that obtained by SDM.

If we put $\alpha \longrightarrow 1, \beta \longrightarrow 1$, and $\varepsilon \longrightarrow 1$ in Eq. (5.2), we reproduce the solution of the problem as follows

$$
\begin{aligned}
& u(x, y, t)=e^{x+y}\left(1-t+\frac{t^{2}}{2 !}-\cdots\right), \\
& v(x, y, t)=e^{x-y}\left(1+t+\frac{t^{2}}{2 !}+\cdots\right), \\
& w(x, y, t)=e^{y-x}\left(1+t+\frac{t^{2}}{2 !}+\cdots\right) .
\end{aligned}
$$

This solution is equivalent to the exact solution in closed form:

$$
u(x, y, t)=e^{x+y-t}, \quad v(x, y, t)=e^{x-y+t}, \quad w(x, y, t)=e^{y-x+t} .
$$

\section{Conclusions}

In this work, the DJM and SDM have been successfully applied to finding the approximate solution of nonlinear system of partial differential equations with Caputo-Fabrizio fractional operator. The example shows that the results of DJM are in excellent agreement with the results given by SDM. The methods are very powerful and efficient in finding analytical as well as numerical solutions for wide classes of linear and non-linear fractional differential equations. 


\section{References}

[1] M. Al-Refai, K. Pal, New Aspects of Caputo-Fabrizio Fractional Derivative, Progr. Fract. Differ. Appl., 5 (2019), 157-166. 2.1, 2.2

[2] A. Atangana, Non validity of index law in fractional calculus: a fractional differential operator with Markovian and nonMarkovian properties, Phys. A, Stat. Mech. Appl., 505 (2018), 688-706. 1

[3] A. Atangana, J. F. Gómez-Aguilar, Fractional derivatives with no-index law property: application to chaos and statistics, Chaos Solitons Fractals, 114 (2018), 516-535. 1

[4] D. Baleanu, H. K. Jassim, A Modification Fractional Homotopy Perturbation Method for Solving Helmholtz and Coupled Helmholtz Equations on Cantor Sets, Fractal Fract., 3 (2019), 8 pages. 1

[5] D. Baleanu, H. K. Jassim, M. Al Qurashi, Approximate Analytical Solutions of Goursat Problem within Local Fractional Operators, J. Nonlinear Sci. Appl., 9 (2016), 4829-4837. 1

[6] D. Baleanu, H. K. Jassim, M. Al Qurashi, Solving Helmholtz Equation with Local Fractional Derivative Operators, Fractal Fract., 3 (2019), 1-13. 1

[7] D. Baleanu, H. K. Jassim, H. Khan, A Modification Fractional Variational Iteration Method for solving Nonlinear Gas Dynamic and Coupled KdV Equations Involving Local Fractional Operators, Therm. Sci., 22 (2018), S165-S175. 1

[8] M. Caputo, M. Fabrizio, A new Definition of Fractional Derivative without Singular Kernel, Progr. Fract. Differ. Appl., 1 (2015), 73-85. 2.1, 2.2

[9] J. F. Gómez-Aguilar, H. Yépez-Martínez, J. Torres-Jiménez, T. Córdova-Fraga, R. F. Escobar-Jiménez, V. H. Olivares-Peregrino, Homotopy perturbation transform method for nonlinear differential equations involving to fractional operator with exponential kernel, Adv. Difference Equ., 2017 (2017), 18 pages. 1, 2.1

[10] H. Jafari, M. Ghorbani, S. Ghasempour, A note on exact solutions for nonlinear integral equations by a modified homotopy perturbation method, New Trends Math. Sci., 2013 (2013), 22-26. 1

[11] H. Jafari, H. K. Jassim, M. Al Qurashi, D. Baleanu, On the Existence and Uniqueness of Solutions for Local differential equations, Entropy, 18 (2016), 1-9. 1

[12] H. Jafari, H. K. Jassim, S. P. Moshokoa, V. M. Ariyan, F. Tchier, Reduced differential transform method for partial differential equations within local fractional derivative operators, Adv. Mech. Eng., 8 (2016), 1-6. 1

[13] H. Jafari, H. K. Jassim, F. Tchier, D. Baleanu, On the Approximate Solutions of Local Fractional Differential Equations with Local Fractional Operators, Entropy, 18 (2016), 1-12. 1

[14] H. Jafari, H. K. Jassim, J. Vahidi, Reduced Differential Transform and Variational Iteration Methods for 3D Diffusion Model in Fractal Heat Transfer within Local Fractional Operators, Therm. Sci., 22 (2018), S301-S307. 1

[15] H. K. Jassim, New Approaches for Solving Fokker Planck Equation on Cantor Sets within Local Fractional Operators, J. Math., 2015 (2015), 1-8. 1

[16] H. K. Jassim, The Approximate Solutions of Three-Dimensional Diffusion and Wave Equations within Local Fractional Derivative Operator, Abstr. Appl. Anal., 2016 (2016), 5 pages. 1

[17] H. K. Jassim, Analytical Approximate Solutions for Local Fractional Wave Equations, Math. Method. Appl. Sci., 43 (2020), 939-947. 1

[18] H. K. Jassim, D. Baleanu, A novel approach for Korteweg-de Vries equation of fractional order, J. Appl. Comput. Mech., 5 (2019), 192-198. 1

[19] H. K. Jassim, M. G. Mohammed, S. A. Khafif, The Approximate solutions of time-fractional Burgers and coupled timefractional Burgers equations, Int. J. Adv. Appl. Math. Mech., 6 (2019), 64-70. 1

[20] H. K. Jassim, W. A. Shahab, Fractional variational iteration method for solving the hyperbolic telegraph equation, J. Phys. Conf. Ser., 1032 (2018), 1-9. 1

[21] D. Kumar, J. Singh, S. Kumar, Analytic and approximate solutions of space-time fractional telegraph equations via Laplace transform, Walailak J. Sci. Technol., 11 (2013), 711-728. 1

[22] I. Podlubny, Fractional Differential Equations, Mathematics in Science and Engineering, Academic Press, USA, (1999). 1

[23] K. Sayevand, H. Jafari, On systems of nonlinear equations: some modified iteration formulas by the homotopy perturbation method with accelerated fourth-and fifth-order convergence, Appl. Math. Model., 40 (2016), 1467-1476. 1

[24] A. Shaikh, A. Tassaddiq, K. S. Nisar, D. Baleanu, Analysis of differential equations involving Caputo-Fabrizio fractional operator and its applications to reaction-diffusion equations, Adv. Difference Equ., 2019 (2019), 14 pages. 1, 2.1

[25] J. Singh, H. K. Jassim, D. Kumar, An efficient computational technique for local fractional Fokker-Planck equation, Phys. A, Stat. Mech. Appl., 555 (2020), 8 pages. 1

[26] S. Xu, X. Ling, Y. Zhao, H. K. Jassim, A Novel Schedule for Solving the Two-Dimensional Diffusion in Fractal Heat Transfer, Therm. Sci., 19 (2015), S99-S103. 1

[27] S. P. Yan, H. Jafari, H. K. Jassim, Local Fractional Adomian Decomposition and Function Decomposition Methods for Solving Laplace Equation within Local Fractional Operators, Adv. Math. Phys., 2014 (2014), 7 pages. 1 